\title{
On Pseudo-Petrov Symmetric Riemannian Manifolds
}

\author{
Sanjib Kumar Jana, ${ }^{1}$ Fusun Nurcan, ${ }^{2}$ Amit Kumar Debnath, ${ }^{3}$ and Joydeep Sengupta ${ }^{3}$ \\ ${ }^{1}$ Department of Mathematics, Balurghat College, Balurghat, Dakshin Dinajpur, West Bengal 733 101, India \\ ${ }^{2}$ Department of Mathematics, Marmara University, Goztepe Campus, 34722 Istanbul, Turkey \\ ${ }^{3}$ Department of Mathematics, University of Gour Banga, Mokdumpur, Malda, West Bengal 732 103, India
}

Correspondence should be addressed to Fusun Nurcan; funal@marmara.edu.tr

Received 29 November 2015; Accepted 16 February 2016

Academic Editor: Luigi C. Berselli

Copyright (C) 2016 Sanjib Kumar Jana et al. This is an open access article distributed under the Creative Commons Attribution License, which permits unrestricted use, distribution, and reproduction in any medium, provided the original work is properly cited.

The present paper deals with pseudo-Petrov symmetric Riemannian manifolds whose space-matter tensor satisfies a special condition. Firstly, basic results of pseudo-Petrov symmetric Riemannian manifolds are obtained. Then, pseudo-Petrov symmetric manifolds which are Einstein, quasi-Einstein, and locally decomposable are examined and some theorems involving these manifolds are proved. Finally, two examples proving the existence of pseudo-Petrov symmetric Riemannian manifolds are given.

\section{Introduction}

In the sense of Chaki, a nonflat Riemannian manifold is said to be pseudosymmetric [1] if its curvature tensor satisfies the following relation:

$$
\begin{aligned}
\left(\nabla_{X} R\right)(Y, Z, U, V)= & 2 \alpha(X) R(Y, Z, U, V) \\
& +\alpha(Y) R(X, Z, U, V) \\
& +\alpha(Z) R(Y, X, U, V) \\
& +\alpha(U) R(Y, Z, X, V) \\
& +\alpha(V) R(Y, Z, U, X)
\end{aligned}
$$

for all vector fields $X, Y, Z, U, V \in \chi\left(M^{n}\right)$, where $\alpha$ is a nonzero 1-form, $\chi\left(M^{n}\right)$ denotes the Lie algebra of all smooth vector fields [over $C^{\infty}(M)$ ] on manifold $M$, and $\nabla$ denotes the operator of covariant differentiation with respect to the metric tensor $g$. The 1 -form $\alpha$ is called the associated 1 -form of the manifold and an $n$-dimensional manifold of this kind is denoted by (PS) $)_{n}$.

In 1949, Petrov [2] introduced a tensor field $P$ of type $(0,4)$ and defined it by

$$
P=R+\frac{k}{2} g \wedge T-\sigma G
$$

where $R$ is the Riemannian curvature tensor of type $(0,4)$, $T$ is the energy-momentum tensor of type $(0,2), k$ is a cosmological constant, $\sigma$ is the energy density (scalar), $G$ is a tensor of type $(0,4)$ given by

$$
G(X, Y, Z, U)=g(X, U) g(Y, Z)-g(X, Z) g(Y, U)
$$

for all $X, Y, Z, U \in \chi(M), \chi(M)$ being the Lie algebra of smooth vector fields on $M$, and the Kulkarni-Nomizu product [3] $E \wedge F$ of two $(0,2)$ tensors $E$ and $F$ is defined by

$$
\begin{aligned}
&(E \wedge F)\left(X_{1}, X_{2}, X_{3}, X_{4}\right) \\
&=E\left(X_{1}, X_{4}\right) F\left(X_{2}, X_{3}\right)+E\left(X_{2}, X_{3}\right) F\left(X_{1}, X_{4}\right) \\
&-E\left(X_{1}, X_{3}\right) F\left(X_{2}, X_{4}\right) \\
&-E\left(X_{2}, X_{4}\right) F\left(X_{1}, X_{3}\right),
\end{aligned}
$$

for $X_{i} \in \chi(M), i=1,2,3,4$. The tensor $P$ is known as the space-matter tensor of type $(0,4)$ of manifold $M$.

Einstein's field equation with cosmological constant [4] is given by

$$
k T=S+\left(\lambda-\frac{r}{2}\right) g,
$$


where $\lambda$ is a cosmological constant, $r$ is the scalar curvature, and $S$ is the Ricci tensor of type $(0,2)$. By virtue of $(5),(2)$ takes the form

$$
P=R+\frac{1}{2}(g \wedge S)-\left(\sigma-\lambda+\frac{r}{2}\right) G .
$$

The present paper deals with a Riemannian manifold $\left(M^{n}, g\right)(n>3)$ whose space-matter tensor $P$ (nonvanishing identically) satisfies the condition

$$
\begin{aligned}
\left(\nabla_{X} P\right)(Y, Z, U, V)= & 2 A(X) P(Y, Z, U, V) \\
& +A(Y) P(X, Z, U, V) \\
& +A(Z) P(Y, X, U, V) \\
& +A(U) P(Y, Z, X, V) \\
& +A(V) P(Y, Z, U, X),
\end{aligned}
$$

where $A$ is a nonzero 1 -form such that $A(X)=g(X, \rho)$ for all $X$. Such a manifold will be called a pseudo-Petrov symmetric manifold and denoted by (PPS $)_{n}$.

In Section 2, we go through some basic results of (PPS) [5]. In Section 3, we study Einstein (PPS $)_{n}$ and prove that scalar curvature can not be vanished in such a manifold. Section 4 is concerned about quasi-Einstein (PPS) ${ }_{n}$ where we discuss $(\mathrm{PPS})_{n}$ which is locally decomposable in Section 5. Finally, the last section deals with nontrivial examples of $(\mathrm{PPS})_{n}$ and also of quasi-Einstein $(\mathrm{PPS})_{n}$.

\section{Some Basic Results of $(\mathrm{PPS})_{n}$}

In this section, we deal with some basic results of (PPS $)_{n}$. Let $\left\{e_{i}: i=1,2, \ldots, n\right\}$ be an orthonormal basis of the tangent space at any point of the manifold. Then, the Ricci tensor $S$ of type $(0,2)$ and the scalar curvature $r$ are given by

$$
\begin{gathered}
S(X, Y)=g(Q X, Y)=\sum_{i=1}^{n} R\left(e_{i}, X, Y, e_{i}\right), \\
r=\sum_{i=1}^{n} S\left(e_{i}, e_{i}\right)=\sum_{i=1}^{n} g\left(Q e_{i}, e_{i}\right),
\end{gathered}
$$

where $Q$ is the symmetric endomorphism corresponding to the Ricci tensor S. From (6), we obtain

$$
\begin{aligned}
& \sum_{i=1}^{n} P\left(e_{i}, Z, U, e_{i}\right) \\
& \quad=\frac{n}{2} S(Z, U)-\left[\frac{n-2}{2} r+(n-1)(\sigma-\lambda)\right] g(Z, U)
\end{aligned}
$$

for all $Z$ and $U$. Differentiating the above equation covariantly with respect to $X$, we get

$$
\begin{aligned}
\sum_{i=1}^{n}( & \left.\nabla_{X} P\right)\left(e_{i}, Z, U, e_{i}\right) \\
= & \frac{n}{2}\left(\nabla_{X} S\right)(Z, U) \\
& -\left[\frac{n-2}{2} d r(X)+(n-1) d \sigma(X)\right] g(Z, U)
\end{aligned}
$$

for all $X, Z$, and $U$. Then, using (6) in (7) and contracting with respect to $Y$ and $V$, we obtain, by virtue of (10), that

$$
\begin{aligned}
n( & \left.\nabla_{X} S\right)(Z, U)-[(n-2) d r(X)+2(n-1) d \sigma(X)] \\
\cdot & g(Z, U)=n[2 A(X) S(Z, U)+A(Z) S(X, U) \\
+ & A(U) S(Z, X)]-\{(n-2) r-2(n-1)(\lambda-\sigma)\} \\
\cdot & {[2 A(X) g(Z, U)+A(Z) g(X, U)} \\
& +A(U) g(Z, X)]+4 P(X, U, Z, \rho) .
\end{aligned}
$$

Setting $Z=U=e_{i}$ in (11) and taking summation over $i, 1 \leq$ $i \leq n$, we get

$$
\begin{aligned}
& A(Q X) \\
& \quad-\frac{1}{n}\left[\frac{1}{2}\left(n^{2}-n-4\right) r-(n+2)(n-1)(\lambda-\sigma)\right] \\
& \quad \cdot A(X)=\frac{3-n}{4} d r(X)-\frac{n-1}{2} d \sigma(X) .
\end{aligned}
$$

Assume that the scalar curvature $r$ and the energy density $\sigma$ satisfy the relation

$$
(n-3) r-2(n-1)(\lambda-\sigma)=0 .
$$

Then, (12) reduces to

$$
\begin{aligned}
A(Q X) & =\frac{2(n-1)}{n(n-3)}(\lambda-\sigma) A(X), \\
\text { that is, } S(X, \rho) & =\frac{2(n-1)}{n(n-3)}(\lambda-\sigma) g(X, \rho) .
\end{aligned}
$$

From (5) and (15), we obtain

$$
T(X, \rho)=\frac{(n-1)(n-2) \sigma-2 \lambda}{k n(n-3)} g(X, \rho) .
$$

This leads to the following theorem.

Theorem 1. In a $(P P S)_{n}$ admitting Einstein's field equation, $((n-1)(n-2) \sigma-2 \lambda) / k n(n-3)$ is an eigenvalue of the energymomentum tensor $T$ corresponding to the eigenvector $\rho$ defined by $g(X, \rho)=A(X)$ for all $X$, provided that relation (13) holds.

\section{Einstein $(P P S)_{n}$}

This section is concerned about a (PPS $)_{n}(n>3)$ which is also an Einstein manifold. The Ricci tensor of Einstein manifold [3] satisfies the condition

$$
S(X, Y)=\frac{r}{n} g(X, Y)
$$

from which it follows that

$$
\begin{array}{r}
d r(X)=0, \\
\left(\nabla_{Z} S\right)(X, Y)=0
\end{array}
$$


In view of (17), (18), and (6), we find that

$$
\begin{aligned}
\left(\nabla_{X} P\right)(Y, Z, U, V)= & \left(\nabla_{X} R\right)(Y, Z, U, V) \\
& -d \sigma(X) G(Y, Z, U, V) .
\end{aligned}
$$

By virtue of (17), (19), and (7), we get

$$
\begin{aligned}
& \left(\nabla_{X} R\right)(Y, Z, U, V)-d \sigma(X) G(Y, Z, U, V)=2 A(X) \\
& \cdot R(Y, Z, U, V)+A(Y) R(X, Z, U, V)+A(Z) \\
& \cdot R(Y, X, U, V)+A(U) R(Y, Z, X, V)+A(V) \\
& \cdot R(Y, Z, U, X)+\left[\lambda-\sigma-\frac{(n-2) r}{2 n}\right] \\
& \cdot[2 A(X) G(Y, Z, U, V)+A(Y) G(X, Z, U, V) \\
& +A(Z) G(Y, X, U, V)+A(U) G(Y, Z, X, V) \\
& +A(V) G(Y, Z, U, X)] .
\end{aligned}
$$

Setting $Y=V=e_{i}$ in (20) and then taking summation over $i$, $1 \leq i \leq n$, we find that

$$
\begin{aligned}
& (n-1) d \sigma(X) g(Z, U)=\left[\frac{(n-2) r}{2 n}-(\lambda-\sigma)\right][(n \\
& -1)\{2 A(X) g(Z, U)+A(Z) g(X, U) \\
& +A(U) g(Z, X)\}+A(X) g(Z, U)-A(Z) \\
& \quad \cdot g(X, U)+A(X) g(Z, U)-A(U) g(X, Z)] \\
& \quad-\frac{r}{n}[2 A(X) g(Z, U)+A(Z) g(X, U)+A(U) \\
& \quad \cdot g(Z, X)]-2 R(X, Z, U, \rho)
\end{aligned}
$$

by virtue of (18). Replacing $Z$ and $U$ by $e_{i}$ in (21) and taking summation over $i, 1 \leq i \leq n$, we have

$$
\begin{aligned}
& n(n-1) d \sigma(X) \\
& \quad=(n+2)[(n-3) r-2(n-1)(\lambda-\sigma)] A(X) .
\end{aligned}
$$

Then, substituting $X$ and $Z$ by $e_{i}$ in (21) and taking summation over $i, 1 \leq i \leq n$, we obtain

$$
\begin{aligned}
& 2(n-1) d \sigma(X) \\
& \quad=(n+2)[(n-3) r-2(n-1)(\lambda-\sigma)] A(X) .
\end{aligned}
$$

By virtue of (22) and (23), we have

$$
(n-3) r-2(n-1)(\lambda-\sigma)=0 .
$$

Finally, in the view of (18) and (24), we get

$$
d \sigma(X)=0 \quad \forall X \text { that is, } \sigma \text { is constant. }
$$

Hence, we can state the following theorem.

Theorem 2. In an Einstein $(P P S)_{n}$ admitting Einstein's field equation, the energy density scalar is constant and it is connected with the scalar curvature by the relation $(n-3) r-$ $2(n-1)(\lambda-\sigma)=0$.
Now, in an Einstein (PPS $)_{n}$ admitting Einstein's field equation, if possible, let the energy density scalar $\sigma$ be equal to the cosmological constant $\lambda$. From (24), it follows that the scalar curvature $r$ vanishes identically. With the help of (20), we find that

$$
\begin{aligned}
\left(\nabla_{X} R\right)(Y, Z, U, V)= & 2 A(X) R(Y, Z, U, V) \\
& +A(Y) R(X, Z, U, V) \\
& +A(Z) R(Y, X, U, V) \\
& +A(U) R(Y, Z, X, V) \\
& +A(V) R(Y, Z, U, X)
\end{aligned}
$$

which shows that the manifold is a $(\mathrm{PS})_{n}$. This leads to a contradiction, as an Einstein manifold can not accommodate a pseudosymmetric structure [1]. So our assumption that $\sigma=$ $\lambda$ is not possible. Hence, we can state the following theorem.

Theorem 3. In an Einstein $(P P S)_{n}$ admitting Einstein's field equation, the energy density satisfies the relation $\sigma \neq \lambda$ and the scalar curvature can not be vanished identically.

Definition 4. A vector field $V$ on a Riemannian manifold is said to be concurrent [6] if and only if there exists a nonzero constant $k$ such that $\nabla_{X} V=k X$ for all $X \in \chi(M)$.

$V$ is said to be parallel, in case $\nabla_{X} V=0$ for all $X \in \chi(M)$.

Let us consider that an Einstein (PPS $)_{n}$ in which the vector field $\rho$ is defined by $g(X, \rho)=A(X)$ is parallel. Then, we have

$$
\nabla_{X} \rho=0 \quad \forall X
$$

If $\rho$ is concurrent, we have

$$
\nabla_{X} \rho=\kappa X, \text { where } \kappa \text { is a constant. }
$$

Therefore, by using Ricci identity, we get $R(X, Y, \rho, U)=0$ which yields

$$
S(Y, \rho)=0
$$

From (17) and (29), it follows that

$$
r=0 \quad \text { since }\|\rho\|=1
$$

which is not possible according to our previous theorem. So our assumption is not true; that is, $\rho$ can not be a concurrent vector field. This leads to the following theorem.

Theorem 5. In an Einstein $(P P S)_{n}$ admitting Einstein's field equation, the vector field $\rho$ defined by $g(X, \rho)=A(X)$ cannot be concurrent.

\section{Quasi-Einstein (PPS) ${ }_{n}$}

In this section, we discuss a quasi-Einstein manifold admitting a pseudosymmetric space-matter tensor. A Riemannian 
manifold $\left(M^{n}, g\right)(n>3)$ is said to be quasi-Einstein [7] if its Ricci tensor $S$ is not identically zero and satisfies that

$$
S=\alpha g+\beta L \otimes L,
$$

where $\alpha, \beta(\neq 0)$ are associated scalars and $L$ is a nonzero 1 form which is defined by $g(X, \varsigma)=L(X)$ for any vector field $X$, with $\varsigma$ being a unit vector field, called the generator of the manifold. Relation (31) implies

$$
r=n \alpha+\beta .
$$

Differentiating covariantly (31) with respect to $X$, we obtain

$$
\begin{aligned}
& \left(\nabla_{X} S\right)(Z, U) \\
& =d \alpha(X) g(Z, U)+d \beta(X) L(Z) L(U) \\
& \quad+\beta\left[\left(\nabla_{X}\right) L(Z) L(U)+\left(\nabla_{X}\right) L(U) L(Z)\right] .
\end{aligned}
$$

In view of (33), (11) takes the following form:

$$
\begin{aligned}
n[ & d \alpha(X) g(Z, U)+d \beta(X) L(Z) L(U) \\
& \left.+\beta\left\{\left(\nabla_{X}\right) L(Z) L(U)+\left(\nabla_{X}\right) L(U) L(Z)\right\}\right] \\
& -[(n-2)\{n d \alpha(X)+d \beta(X)\}+2(n-1) d \sigma(X)] \\
& \cdot g(Z, U)=n[2 A(X) S(Z, U)+A(Z) S(X, U) \\
& +A(U) S(Z, X)]-\{(n-2) r-2(n-1)(\lambda-\sigma)\} \\
& \cdot[2 A(X) g(Z, U)+A(Z) g(X, U) \\
& +A(U) g(Z, X)]+4 P(X, U, Z, \rho) .
\end{aligned}
$$

Firstly, taking contraction of (34) with respect to $Z$ and $U$, we have

$$
\begin{aligned}
& n(n-3)[n d \alpha(X)+d \beta(X)]+2 n(n-1) d \sigma(X) \\
& \quad+4 n A(Q X)=2\left[\left(n^{2}-n-4\right)(n \alpha+\beta)\right. \\
& \quad+2(n+2)(n-1)(\sigma-\lambda)] A(X) .
\end{aligned}
$$

Then, also contracting (34) with respect to $X$ and $U$, we have

$$
\begin{aligned}
& (n-4)[n d \alpha(X)+d \beta(X)]+4(n-1) d \sigma(X) \\
& \quad+4 n A(Q X)=2\left[\left(n^{2}-n-4\right)(n \alpha+\beta)\right. \\
& \quad+2(n+2)(n-1)(\sigma-\lambda)] A(X) .
\end{aligned}
$$

Thus, (35) and (36) yield

$$
(n-2)[n d \alpha(X)+d \beta(X)]-2(n-1) d \sigma(X)=0 .
$$

Hence, we can state the following theorem.

Theorem 6. In a quasi-Einstein $(P P S)_{n}$ admitting Einstein's field equation, the scalars $\alpha$ and $\beta$ and the energy density $\sigma$ are connected by relation (37).
Now, from (37), it is clear that $\sigma$ will be also constant if $r$ is constant; that is, from (36), we have that

$$
\begin{aligned}
& A(Q X)=\frac{1}{2 n}\left[\left(n^{2}-n-4\right)(n \alpha+\beta)\right. \\
& \quad+2(n+2)(n-1)(\sigma-\lambda)] A(X)
\end{aligned}
$$

which implies

$$
\begin{aligned}
& S(X, \rho)=\frac{1}{2 n}\left[\left(n^{2}-n-4\right)(n \alpha+\beta)\right. \\
& \quad+2(n+2)(n-1)(\sigma-\lambda)] g(X, \rho) .
\end{aligned}
$$

So, by the above relation and (5), we obtain

$$
\begin{aligned}
& T(X, \rho)=\frac{1}{4 n k}\left[\left(n^{2}-n-9\right)(n \alpha+\beta)\right. \\
& \left.\quad+4(n+2)(n-1) \sigma-2\left(2 n^{2}+2 n-5\right) \lambda\right] g(X, \rho) .
\end{aligned}
$$

Thus, we get the following theorem.

Theorem 7. In a quasi-Einstein $(P P S)_{n}$ admitting Einstein's field equation, $(1 / 4 n k)\left[\left(n^{2}-n-9\right)(n \alpha+\beta)+4(n+2)(n-\right.$ 1) $\left.\sigma-2\left(2 n^{2}+2 n-5\right) \lambda\right]$ is an eigenvalue of the energymomentum tensor $T$ corresponding to the eigenvector $\rho$ defined by $g(X, \rho)=A(X)$ for all $X$.

Let us consider that $\varsigma$ is a concurrent vector field; that is,

$$
\nabla_{X} \varsigma=\kappa X \text {, where } \kappa \text { is a constant. }
$$

Hence, by the Ricci identity, it follows that $R(X, Y, \varsigma, U)=0$ which yields

$$
S(X, \varsigma)=0
$$

From (31) and (42), we have

$$
\alpha+\beta=0 \quad \text { since }\|\varsigma\|=1 .
$$

This leads to the following theorem.

Theorem 8. In a quasi-Einstein $(P P S)_{n}$ admitting Einstein's field equation, if the vector field $\varsigma$ is concurrent then the associated scalars $\alpha$ and $\beta$ are connected by relation (43).

\section{Locally Decomposable $(\mathrm{PPS})_{n}$}

A differentiable manifold is said to be a product manifold [6] if and only if it can be expressed as $M_{1}^{p} \times M_{2}^{n-p}$, where $2 \leq p \leq$ $n-2$. In this case, we say that $M$ admits both a local product structure and a separating coordinates system.

Moreover, if $M$ is a local product Riemannian manifold, then there exists a Riemannian metric given by

$$
d s^{2}=g_{i j} d x^{i} d x^{j}+g_{\alpha \beta} d y^{\alpha} d y^{\beta},
$$

where $x^{i}$ and $y^{\alpha}$ are local coordinates on $M_{1}$ and $M_{2}$, respectively. Here $i$ and $j$ run over $1,2, \ldots, p$ and $\alpha$ and $\beta$ run 
over $p+1, p+2, \ldots, n$. Equivalently, the manifolds $M_{1}$ and $M_{2}$ are orthogonal. Further, if $g_{i j}$ 's are functions of $x^{i}$ s only and $g_{\alpha \beta}$ 's are functions of $y^{\alpha}$ 's only, then such a manifold is called a locally decomposable Riemannian manifold. For a locally decomposable Riemannian manifold, we have

$$
\begin{aligned}
& \Gamma_{\alpha \beta}^{i}=0=\Gamma_{j \beta}^{i}, \\
& \Gamma_{i j}^{\alpha}=0=\Gamma_{i \beta}^{\alpha},
\end{aligned}
$$

for all $i$ and $j$ and $\alpha$ and $\beta$. Let $\left(M^{n}, g\right)$ be a locally decomposable Riemannian manifold with a separating coordinates system such that we can express $M^{n}=M_{1}^{p} \times M_{2}^{n-p}(2 \leq p \leq$ $n-2)$. Then, the relations in (45) hold. We further assume that $M$ is a (PPS) ${ }_{n}$; that is, there exists a smooth nonzero 1 -form $A$ such that

$$
\begin{aligned}
\left(\nabla_{X} P\right)(Y, Z, U, V)= & 2 A(X) P(Y, Z, U, V) \\
& +A(Y) P(X, Z, U, V) \\
& +A(Z) P(Y, X, U, V) \\
& +A(U) P(Y, Z, X, V) \\
& +A(V) P(Y, Z, U, X),
\end{aligned}
$$

for all $X, Y, Z, U, V \in \chi(M)$. Then, we find the following relations:

$$
\begin{aligned}
& \left(\nabla_{\bar{X}} P\right)(\bar{Y}, \bar{Z}, \bar{U}, \bar{V})=2 A(\bar{X}) P(\bar{Y}, \bar{Z}, \bar{U}, \bar{V}) \\
& \text { + } A(\bar{Y}) P(\bar{X}, \bar{Z}, \bar{U}, \bar{V})+A(\bar{Z}) P(\bar{Y}, \bar{X}, \bar{U}, \bar{V}) \\
& +A(\bar{U}) P(\bar{Y}, \bar{Z}, \bar{X}, \bar{V})+A(\bar{V}) P(\bar{Y}, \bar{Z}, \bar{U}, \bar{X}), \\
& A(\stackrel{*}{X}) P(\bar{Y}, \bar{Z}, \bar{U}, \bar{V})=0 \text {, } \\
& A(\bar{Y}) P(\stackrel{*}{X}, \bar{Z}, \bar{V}, \stackrel{*}{U})-A(\bar{Z}) P(\stackrel{*}{X}, \bar{Y}, \bar{V}, \stackrel{*}{U}) \\
& +A(\bar{V}) P\left(\stackrel{*}{X}, U^{U}, \bar{Y}, \bar{Z}\right)=0, \\
& \left(\nabla_{\bar{X}} P\right)(\stackrel{*}{Y}, \bar{Z}, \bar{U}, \stackrel{*}{V})=2 A(\bar{X}) P\left(\stackrel{Y}{Y}^{*} \bar{Z}, \bar{U}, \stackrel{*}{V}\right) \\
& +A(\bar{Z}) P(\stackrel{*}{Y}, \bar{X}, \bar{U}, \stackrel{*}{V})+A(\bar{U}) P(\stackrel{*}{Y}, \bar{Z}, \bar{X}, \stackrel{*}{V}), \\
& \left(\nabla_{X}^{*} P\right)(\stackrel{*}{Y}, \bar{Z}, \bar{U}, \stackrel{*}{V})=2 A(\stackrel{*}{X}) P(\stackrel{*}{Y}, \bar{Z}, \bar{U}, \stackrel{*}{V}) \\
& +A(\stackrel{*}{Y}) P(\stackrel{*}{X}, \bar{Z}, \bar{U}, \stackrel{*}{V})+A(\stackrel{*}{V}) P(\stackrel{*}{Y}, \bar{Z}, \bar{U}, \stackrel{*}{X}), \\
& A(\stackrel{*}{Y}) P(\stackrel{*}{Z}, \bar{X}, \bar{U}, \stackrel{*}{V})-A(\stackrel{*}{Z}) P(\stackrel{*}{Y}, \bar{X}, \bar{U}, \stackrel{*}{V}) \\
& +A(\stackrel{*}{V}) P(\stackrel{*}{Y}, \stackrel{*}{Z}, \bar{X}, \bar{U})=0, \\
& A(\bar{X}) P(\stackrel{*}{Y}, \stackrel{*}{Z}, \stackrel{*}{U}, \stackrel{*}{V})=0 \text {, }
\end{aligned}
$$

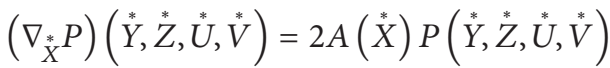

$$
\begin{aligned}
& +A(\stackrel{*}{Y}) P\left(\stackrel{*}{X}, \stackrel{*}{Z}, \stackrel{*}{U}^{\prime}, \stackrel{*}{V}\right)+A(\stackrel{*}{Z}) P(\stackrel{*}{Y}, \stackrel{*}{X}, \stackrel{*}{U}, \stackrel{*}{V})
\end{aligned}
$$

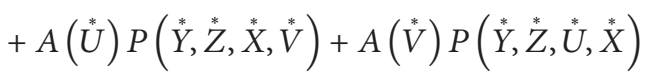

for $\bar{X}, \bar{Y}, \bar{Z}, \bar{U}, \bar{V} \in \chi\left(M_{1}\right)$ and $\stackrel{*}{X}, \stackrel{*}{Y}, \stackrel{*}{Z}, \stackrel{*}{U}, \stackrel{*}{V} \in \chi\left(M_{2}\right)$. From (48), we consider two cases, namely,

(1) $A=0$ on $M_{2}$;

(2) $P=0$ in $M_{1}$.

At first, we consider case (1): from (51), it follows that

$$
\left(\nabla_{X}^{*} P\right)(\stackrel{*}{Y}, \bar{Z}, \bar{U}, \stackrel{*}{V})=0
$$

which implies

$$
\left(\nabla_{\dot{X}}^{*} S\right)(\stackrel{*}{Y}, \stackrel{*}{V})=\left(\stackrel{*}{X} r+2 \stackrel{*}{X} \sigma^{\prime}\right) g\left({ }_{Y}^{*}, \stackrel{*}{V}\right) .
$$

Also, from (54), we obtain

$$
\begin{aligned}
& \left(\nabla_{X}^{*} P\right)\left(\stackrel{*}{Y}, \stackrel{*}{Z}, \stackrel{*}{U}^{U}, \stackrel{*}{V}\right)=0, \\
& \text { that is, }\left(\nabla_{X}^{*} R\right)\left(\stackrel{*}{Y}, \stackrel{*}{Z}, \stackrel{*}{U}^{*}, \stackrel{*}{V}^{\prime}\right) \\
& +\frac{1}{2}\left\{\left(\nabla_{X}^{*} S\right)\left(*_{Z}^{*}, \stackrel{U}{U}^{*}\right) g(\stackrel{*}{Y}, \stackrel{*}{V})\right.
\end{aligned}
$$

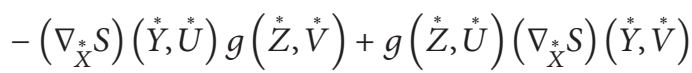

$$
\begin{aligned}
& \left.-g(\stackrel{*}{Y}, \stackrel{*}{U})\left(\nabla_{X}^{*} S\right)(\stackrel{*}{Z}, \stackrel{*}{V})\right\}-\left(\frac{1}{2} \stackrel{*}{X} r+\stackrel{*}{X} \sigma\right)
\end{aligned}
$$

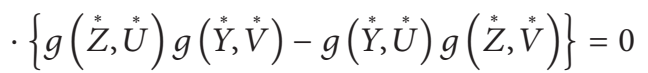

which yields that

$$
\begin{aligned}
& (n-p)\left(\nabla_{X}^{*} S\right)(\stackrel{*}{Y}, V) \\
& =(n-2 p-1)\left(\frac{1}{2} \stackrel{*}{X}^{*}+\stackrel{*}{X}^{*}\right) g(\stackrel{*}{Y}, \stackrel{*}{V}),
\end{aligned}
$$

where the scalar curvature and energy density on $M_{2}$ are denoted by $\stackrel{*}{r}$ and $\stackrel{*}{\sigma}$, respectively. From (56) and (59), it is easy to see that $\stackrel{*}{X}^{*}=\stackrel{*}{X} r$ and $\stackrel{*}{X}^{*}=\stackrel{*}{X} \sigma$ satisfy

$$
\stackrel{*}{X}^{*}+2 \stackrel{*}{X}^{*}=0
$$

In view of (59) and (60), we find that $\left(\nabla_{X}^{*} S\right)\left(Y_{Y}^{*}, V^{*}\right)=0$ for all $\stackrel{*}{X}, \stackrel{*}{Y}$, and $\stackrel{*}{V}$ in $\chi\left(M_{2}\right)$. By virtue of this relation and (60), (58) reduces to

$$
\left(\nabla_{X}^{*} R\right)\left(\stackrel{*}{Y}, \stackrel{*}{Z}, \stackrel{U}{U}^{*}, \stackrel{*}{V}\right)=0
$$


Secondly, we discuss case (2): from $P=0$ in $M_{1}$, we find

$$
\begin{aligned}
& R(\bar{X}, \bar{Y}, \bar{Z}, \bar{U})+\frac{1}{2}[S(\bar{Y}, \bar{Z}) g(\bar{X}, \bar{U}) \\
& \quad-S(\bar{X}, \bar{Z}) g(\bar{Y}, \bar{U})+g(\bar{Y}, \bar{Z}) s(\bar{X}, \bar{U}) \\
& \quad-g(\bar{X}, \bar{Z}) s(\bar{Y}, \bar{U})]-\left(\frac{r}{2}-\lambda+\sigma\right) \\
& \quad \cdot\{g(\bar{Y}, \bar{Z}) g(\bar{X}, \bar{U})-g(\bar{X}, \bar{Z}) g(\bar{Y}, \bar{U})\}=0
\end{aligned}
$$

which yields

$$
p S(\bar{Y}, \bar{Z})+\{\bar{r}-(p-1)(r-2 \lambda+2 \sigma)\} g(\bar{Y}, \bar{Z})=0,
$$

where $\bar{r}$ is the scalar curvature on $M_{1}$. Thus, we find

$$
\bar{r}=(p-1)\left(\frac{1}{2} r-\lambda+\sigma\right) .
$$

Using (64) in (63), we obtain

$$
S(\bar{Y}, \bar{Z})=\frac{\bar{r}}{p} g(\bar{Y}, \bar{Z}) .
$$

By virtue of the last relation, (62) reduces to

$$
R(\bar{X}, \bar{Y}, \bar{Z}, \bar{U})=\frac{\bar{r}}{p(p-1)} G(\bar{X}, \bar{Y}, \bar{Z}, \bar{U})
$$

which shows that the manifold $M_{1}$ is of constant curvature. Hence, we have the following theorem.

Theorem 9. Let $\left(M^{n}, g\right)$ be a locally decomposable Riemannian manifold such that $M=M_{1}^{p} \times M_{2}^{n-p}(2 \leq p \leq n-2)$. If $M$ is a $(P P S)_{n}$, then one gets that

(1) $M_{2}$ is a locally symmetric manifold if $A=0$ on $M_{2}$,

(2) $M_{1}$ is a manifold of constant curvature if $P=0$ in $M_{1}$.

Similarly, in view of (53), we can state the following theorem.

Theorem 10. Let $\left(M^{n}, g\right)$ be a locally decomposable Riemannian manifold such that $M=M_{1}^{p} \times M_{2}^{n-p}(2 \leq p \leq n-2)$. If $M$ is a $(P P S)_{n}$, then one obtains that

(1) $M_{1}$ is a locally symmetric manifold if $A=0$ on $M_{1}$,

(2) $M_{2}$ is of constant curvature if $P=0$ in $M_{2}$.

Now, contracting (49) with respect to $\stackrel{*}{X}$ and $\stackrel{*}{U}$, we obtain

$$
\begin{aligned}
& A(\bar{Y})[\{\stackrel{*}{r g}(\bar{Z}, \bar{V})+(n-p) S(\bar{Z}, \bar{V})\} \\
& -(n-p)(r-2 \lambda+2 \sigma) g(\bar{Z}, \bar{V})]-A(\bar{Z}) \\
& \cdot\left[\left\{{ }^{*} g(\bar{Y}, \bar{V})+(n-p) S(\bar{Y}, \bar{V})\right\}\right. \\
& -(n-p)(r-2 \lambda+2 \sigma) g(\bar{Y}, \bar{V})]=0
\end{aligned}
$$

which yields that

$$
A(Q \bar{Y})=\frac{r_{1}}{n-p} A(\bar{Y})
$$

where

$$
\begin{aligned}
r_{1} & =\frac{1}{n-1}\{(n-p)(n-2 p-1) \bar{r} \\
& -(p-1)(n-2 p+1) \stackrel{*}{r}\} .
\end{aligned}
$$

This result leads to the following theorem.

Theorem 11. Let $\left(M^{n}, g\right)$ be a locally decomposable Riemannian manifold such that $M=M_{1}^{p} \times M_{2}^{n-p}(2 \leq p \leq n-2)$. If $M$ is $a(P P S)_{n}$, then the relation

$$
A(Q \bar{X})=-\frac{r_{1}}{n-p} A(\bar{X})
$$

holds for any vector field $\bar{X}$ on $\chi\left(M_{1}\right)$.

Similarly, from (52), we can state the following theorem.

Theorem 12. Let $\left(M^{n}, g\right)$ be a locally decomposable Riemannian manifold such that $M=M_{1}^{p} \times M_{2}^{n-p}(2 \leq p \leq n-2)$. If $M$ is a (PPS $)_{n}$, then the relation

$$
A(Q \stackrel{*}{X})=-\frac{r_{2}}{p} A(\stackrel{*}{X})
$$

holds for any vector field $\stackrel{*}{X}$ on $\chi\left(M_{2}\right)$.

\section{Some Examples of (PPS)}

In this section, the existence of (PPS $)_{n}$ is proved by means of citing some examples.

Example 1. Let us consider the manifold $M^{4}=\left\{\left(x^{1}, x^{2}, x^{3}\right.\right.$, $\left.\left.x^{4}\right) \in \mathbb{R}^{4}\right\}$ with the metric

$$
\begin{aligned}
d s^{2} & =g_{i j} d x^{i} d x^{j} \\
& =\left(d x^{2}\right)^{2}+2 e^{x^{2}}\left[d x^{1} d x^{2}+d x^{3} d x^{4}\right] .
\end{aligned}
$$

Then, the only nonvanishing components of the curvature tensor, the Ricci tensor, and the scalar curvature are given by

$$
\begin{aligned}
R_{2342} & =-\frac{1}{4} e^{x^{2}} ; \\
S_{22} & =-\frac{1}{2} ; \\
r & =0 .
\end{aligned}
$$

And, furthermore, considering $\sigma=\lambda$, we find only nonvanishing component of the space-matter tensor and its covariant derivative as follows:

$$
\begin{aligned}
P_{2342} & =-\frac{1}{2} e^{x^{2}} \\
P_{2342,2} & =-e^{x^{2}}
\end{aligned}
$$


where ", in between indices denotes covariant differentiation with respect to the coordinates. Let us consider the 1 -form $A$ as

$$
A\left(\frac{\partial}{\partial x^{i}}\right)=A_{i}= \begin{cases}\frac{1}{2} & \text { for } i=2 \\ 0 & \text { otherwise }\end{cases}
$$

In the manifold under consideration, with the help of (75), (7) reduces to the following equation:

$$
P_{2342,2}=4 A_{2} P_{2342}+A_{3} P_{2242}+A_{4} P_{2322} \text {. }
$$

In the cases other than (76), either the result is trivial or both sides vanish identically. Now, we check the validity of the above equation:

$$
\begin{aligned}
& \text { right hand side of }(76)=2 P_{2342}=-e^{x^{2}} \\
& \quad=\text { left hand side of }(76)
\end{aligned}
$$

Since the manifold under consideration is a (PPS) $)_{4}$, we can state the following theorem.

Theorem 13. Let $\left(M^{4}, g\right)$ be a Riemannian manifold endowed with the metric

$$
\begin{array}{r}
d s^{2}=g_{i j} d x^{i} d x^{j}=\left(d x^{2}\right)^{2}+2 e^{x^{2}}\left[d x^{1} d x^{2}+d x^{3} d x^{4}\right] \\
(i, j=1,2,3,4) .
\end{array}
$$

Then, $\left(M^{4}, g\right)$ is a $(P P S)_{4}$ admitting Einstein's field equation of nonvanishing scalar curvature with $\sigma=\lambda$.

Example 2. Let $M^{4}=\left\{\left(x^{1}, x^{2}, x^{3}, x^{4}\right) \in \mathbb{R}^{4}\right\}$ be an open subset of $\mathbb{R}^{4}$ endowed with the metric

$$
\begin{aligned}
d s^{2} & =g_{i j} d x^{i} d x^{j} \\
& =e^{-x^{1}}\left[\left(d x^{1}\right)^{2}-\left(d x^{2}\right)^{2}+2 d x^{3} d x^{4}\right],
\end{aligned}
$$

where $i$ and $j$ run from 1 to 4 . Then, the only nonvanishing components of the curvature tensor, the Ricci tensor, and the scalar curvature are given by

$$
\begin{aligned}
R_{2342} & =-\frac{1}{4} e^{x^{1}}=R_{3434} \\
S_{22} & =-\frac{1}{2} \\
S_{34} & =\frac{1}{2} \\
r & =\frac{3}{2} e^{x^{1}} .
\end{aligned}
$$

We will now verify that $M^{4}$ is a $(\mathrm{QE})_{4}$. To verify this, let us consider that the 1 -form $L$ and associated scalars $\alpha$ and $\beta$ are as follows:

$$
\begin{aligned}
L\left(\frac{\partial}{\partial x^{i}}\right) & =L_{i}= \begin{cases}e^{-x^{1} / 2} & \text { for } i=1, \\
0 & \text { otherwise, }\end{cases} \\
\alpha & =\frac{1}{2} e^{x^{1}} ; \\
\beta & =-\frac{1}{2} e^{x^{1}} .
\end{aligned}
$$

According to the definition of $M^{4}$, (31) reduces to

$$
S_{i i}=\alpha g_{i i}+\beta L_{i} L_{i}, \quad \text { for } i=1,2,3,4,
$$

since the components of (31) vanish identically and relation (31) holds trivially for the cases other than (82). By virtue of (79), (80), and (81), it follows that

$$
\begin{aligned}
& \text { right hand side of }(82)=\frac{1}{2} e^{x^{1}} g_{11}-\frac{1}{2} e^{x^{1}} L_{1} L_{1}=0 \\
& =\text { left hand side of }(82)
\end{aligned}
$$

for $i=1$. By a similar argument, it can be easily shown that relation (82) also holds for $i=2,3,4$. Therefore, $\left(M^{4}, g\right)$ is a $(\mathrm{QE})_{4}$.

Now, considering the energy density function $\sigma$ as a function of $x^{1}$ only and taking its value equal to $\lambda-(1 / 2) e^{x^{1}}$, we calculate only nonvanishing components of the spacematter tensor and its covariant derivatives in the form of

$$
\begin{gathered}
P_{2342}=-\frac{1}{2} e^{-x^{1}}=P_{3434} \\
P_{2342,1}=-\frac{1}{2} e^{x^{1}}=P_{3434,1}
\end{gathered}
$$

where "," denotes the covariant differentiation with respect to the coordinates. Let us take the 1 -form $A$ as follows:

$$
A\left(\frac{\partial}{\partial x^{i}}\right)=A_{i}= \begin{cases}\frac{1}{2} & \text { for } i=1 \\ 0 & \text { otherwise }\end{cases}
$$

In the manifold under consideration, (7) reduces to the following equations by (85):

$$
\begin{aligned}
P_{2342,1}= & 2 A_{1} P_{2342}+A_{2} P_{1342}+A_{3} P_{2142}+A_{4} P_{2312} \\
& +A_{1} P_{2341}, \\
P_{3434,1}= & 2 A_{1} P_{3434}+A_{3} P_{1434}+A_{4} P_{3134}+A_{3} P_{3414} \\
& +A_{4} P_{3431} .
\end{aligned}
$$

In the cases other than (86), either the result is trivial or both sides vanish identically. With the help of (84) and (85), one can easily check the validity of the above relations. Therefore, the manifold under consideration is a (PPS $)_{4}$. Hence, we can state the following theorem. 
Theorem 14. Let $\left(M^{4}, g\right)$ be a Riemannian manifold endowed with the metric

$$
\begin{aligned}
d s^{2} & =g_{i j} d x^{i} d x^{j} \\
& =e^{-x^{1}}\left[\left(d x^{1}\right)^{2}-\left(d x^{2}\right)^{2}+2 d x^{3} d x^{4}\right] \\
& (i, j=1,2,3,4) .
\end{aligned}
$$

Then, $\left(M^{4}, g\right)$ is a $(P P S)_{4}$ admitting Einstein's field equation of nonvanishing scalar curvature with $\sigma=\lambda-(1 / 2) e^{x^{1}}$.

\section{Competing Interests}

The authors declare that there are no competing interests regarding the publication of this paper.

\section{References}

[1] M. C. Chaki, “On pseudosymmetric manifolds," Bulgarian Journal of Physics, vol. 15, pp. 526-531, 1988.

[2] A. Z. Petrov, Einstein Spaces, Pergamon Press, Oxford, UK, 1949.

[3] A. L. Besse, Einstein Manifold, Springer, Berlin, Germany, 1987.

[4] B. O’Neill, Semi-Riemannian Geometry, Academic Press, New York, NY, USA, 1983.

[5] A. A. Shaikh and S. K. Jana, "On weakly quasi-conformally symmetric manifolds," SUT Journal of Mathematics, vol. 43, no. 1, pp. 61-83, 2007.

[6] J. A. Schouten, Ricci-Calculus, Springer, Berlin, Germany, 1954.

[7] M. C. Chaki and R. K. Maity, "On quasi Einstein manifolds," Publicationes Mathematicae Debrecen, vol. 57, no. 3-4, pp. 297306, 2000. 


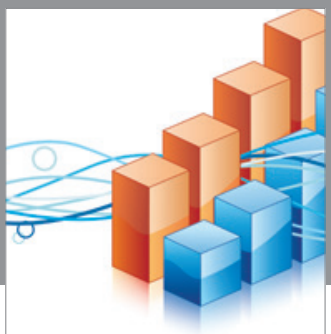

Advances in

Operations Research

vatem alat4

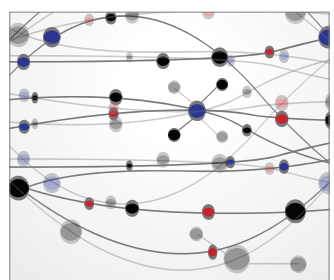

\section{The Scientific} World Journal
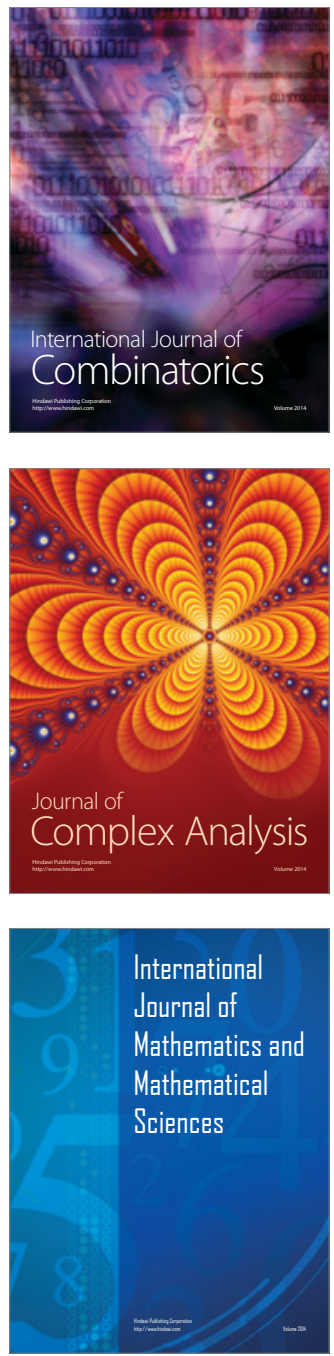
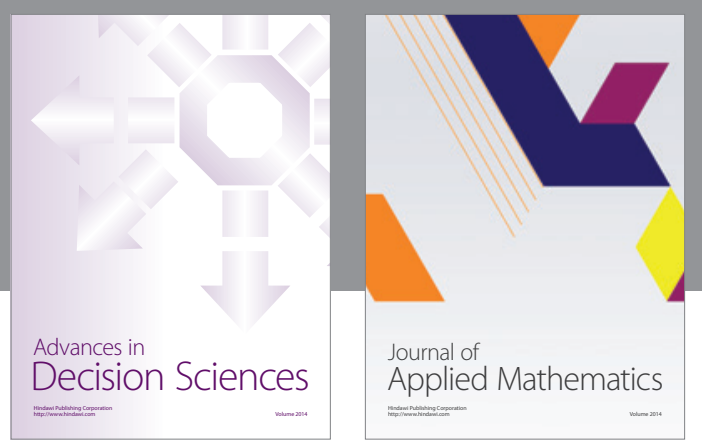

Algebra

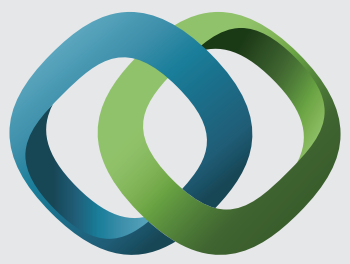

\section{Hindawi}

Submit your manuscripts at

http://www.hindawi.com
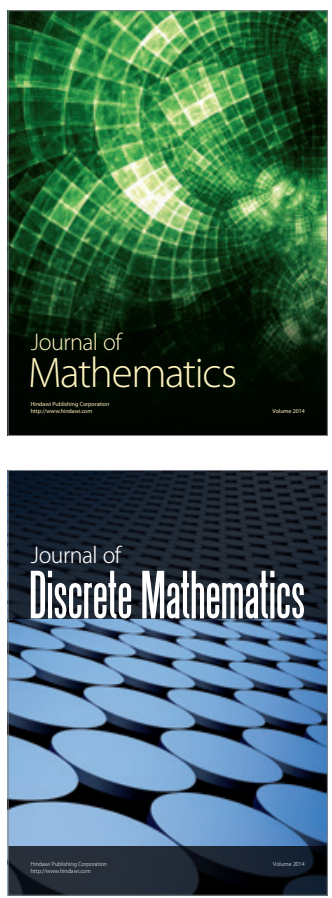

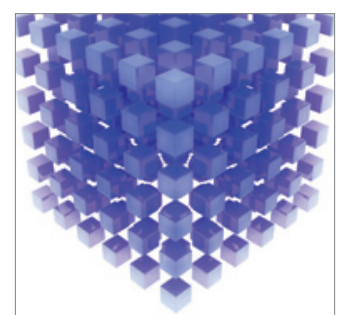

Mathematical Problems in Engineering
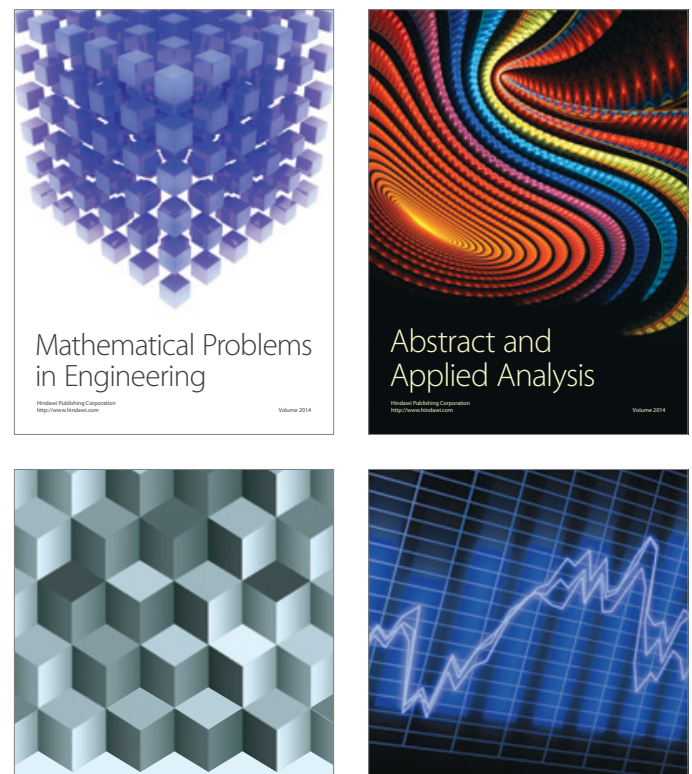

Journal of

Function Spaces

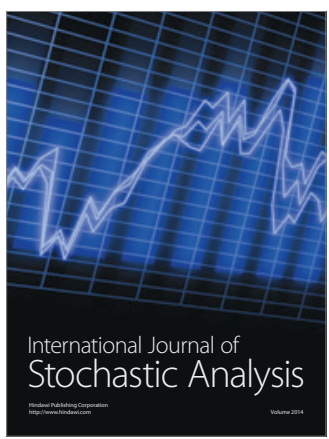

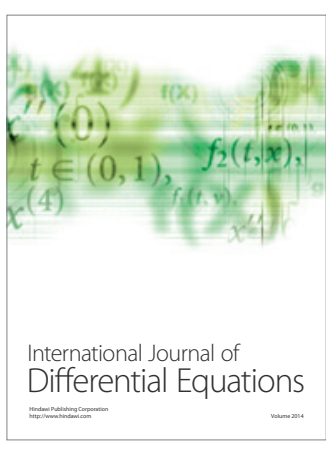
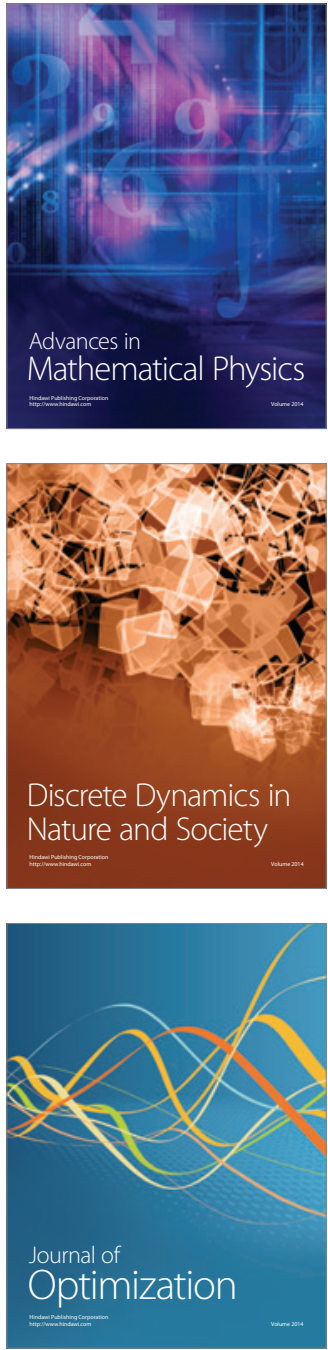